\title{
On the social nature of eyes:
}

\section{The effect of social cues in interaction and individual choice tasks.}

\author{
Aurélien Baillon, Asli Selim, Dennie van Dolder \\ Erasmus School of Economics, Erasmus University Rotterdam
}

Published in Evolution and Human Behavior, March 2013 (34:2), 146-154

\begin{abstract}
In an experimental setting, we applied a dual strategy to better understand the effect of pictures of eyes on human behavior. First, we investigated whether the effect of eyes was limited to interaction tasks in which the subjects' decisions influenced the outcomes of other subjects. We expanded the range of tasks to include individual choice tasks in which the subjects' decisions only influenced their own outcomes. Second, we investigated whether pictures of eyes were one of many social cues or were unique in their effect. We compared the effect of pictures of eyes with the effect of a different condition in which we presented the subjects with pictures of other students (peers). Our results suggest that the effect of pictures of eyes is limited to interaction tasks and that eyes should be considered distinct from other social cues, such as reminders of peers. While pictures of eyes uniformly enhanced pro-social behavior in interaction tasks, this was not the case for reminders of peers. Furthermore, the reminders of peers led to more rational behavior in individual choice tasks, whereas the effect of pictures of eyes was limited to situations involving interaction. Combined, these findings are in line with the claim that the effect of pictures of eyes on behavior is caused by a social exchange heuristic that works to enhance mutual cooperative behavior.
\end{abstract}

JEL: C72, C91, C92, D03, D81.

Version: December 2012

Keywords: Altruism; Ambiguity; Compound lotteries; Cooperation; Cues; Dictator game; Eyes; Generosity; Joy of destruction; Reputation.

Postal address for manuscript correspondence: Erasmus University of Rotterdam, P.O. Box 1738, 3000 DR, Rotterdam, the Netherlands. E: baillon@ese.eur.nl. The authors are grateful to Han Bleichrodt, Rafael Huber, Umut Keskin, Jim Leonhardt, Kirsten Rohde, Joeri Sol, Jan Stoop, Martijn van den Assem and the anonymous reviewers for their many constructive and valuable comments on previous versions of this paper. The paper benefited from discussion with seminar participants at the Erasmus University of Rotterdam, and with participants of the Tiber Symposium on Psychology and Economics 2011 at Tilburg University, the Subjective Probability, Utility, and Decision Making (SPUDM) 2011 conference in Kingston upon Thames, the ErasmusTechnion Workshop on Decisions and Predictions at Ein Bokek, and the Foundations and Applications of Utility, Risk and Decision Theory (FUR) 2012 conference at Georgia State University. We gratefully acknowledge support from the Netherlands Organization of Scientific Research (NWO) and the Tinbergen Institute. 


\section{Introduction}

Humans frequently behave altruistically, even towards genetically unrelated strangers. While some of this altruistic behavior can likely be explained by concerns for the actor's (possible third-party) reputation, it has been argued that this explanation is incomplete. Tightly controlled economic experiments have repeatedly shown that subjects behave in an altruistic manner towards anonymous strangers, even when opportunities for repeated interaction and reputation formation are systematically ruled out (cf. Camerer, 2003). Recent literature, however, has shown that people are sensitive to subtle cues of being watched. In particular, it was demonstrated that, in anonymous experimental settings, the mere presence of pictures of a pair of eyes, or an eye-like stimulus, led to significant increases in donations to strangers in dictator games (Haley \& Fessler, 2005; Oda et al., 2011; Rigdon et al., 2009; Nettle et al., 2013), increased donations to a public good (Burnham \& Hare, 2007), and induced greater disapproval of moral transgressions (Bourrat et al., 2011). The susceptibility of human beings to these subtle cues implies that, even in an anonymous laboratory setting, pro-social behavior should not necessarily be viewed as purely intrinsic (Haley \& Fessler, 2005; Jaeggi et al., 2010).

A number of studies have investigated the generality of the effect of eyes on social behavior and have attempted to gain deeper insight into the possible mechanisms underlying this effect. A potential concern is that the observed phenomenon may have been caused by an experimenter demand effect (Ekström, 2012). Field experiments, however, suggest that this is not the case, as eyelike stimuli have induced pro-social behavior even when the subjects did not know that they were participating in an experiment. Bateson et al. (2006) studied the effect pictures of eyes on the amount of money that employees at a university psychology department contributed to an "honesty box" in the coffee room. The authors found that, when a picture of eyes was placed next to the "honesty box", the employee donations tripled. Ernest-Jones et al. (2011) showed that placing pictures of eyes in a university cafeteria that required diners to clear their own trays halved the odds of littering. However, the effect of eyes was only significant when the cafeteria was relatively quiet. Similarly, Ekström (2012) found that pictures of eyes increased the amount of money that was donated to charity in Swedish supermarkets by $30 \%$ during days on which relatively few people visited the stores. On the days on which the stores were busy, the eyes had no effect on customer donations. Finally, Powell et al. (2012) reported similar results to the previous findings. The authors found that displaying pictures of eyes on charity collection buckets in a supermarket increased donations and that this effect was significantly stronger when the supermarket was quiet rather than busy. 
Although the eye effect appeared to be robust in field settings, several studies suggest that there are conditions under which these effects will not occur. The field studies discussed above suggested that pictures of eyes influence behavior only when the subject is in a non-crowded setting. Fehr and Schneider (2009) found that eyes did not influence the tendency of trustees to repay trust in a trust game. In Mifune et al. (2010), pictures of eyes increased donations in a dictator game when the recipient was an in-group member, but not when the recipient was an out-group member.

The common interpretation of the eye effect is that pictures of eyes trigger feelings of being watched, which in turn activate reputation concerns and subsequent behavioral changes. Such an argument seems plausible, given that actual opportunities to acquire a positive reputation that may pay off in the future have been found to enhance pro-social behavior (Engelmann \& Fischbacher, 2009; Gächter \& Fehr, 1999; Milinski et al., 2001, 2002; Rege \& Telle 2004; Seinen \& Schram 2006; Wedekind \& Milinski 2000). To the best of our knowledge, Oda et al. (2011) provided the only direct test of this conjecture. The authors showed that the eye effect was mediated by expectations of future reward but not by a fear of punishment.

In the present study, we applied a dual strategy to better understand the effect of eyes on human behavior by expanding both the nature of the tasks and the types of social cues that were used as stimuli. Firstly, we examined whether the influence of eyes was limited to interaction tasks in which the subjects' decisions also influenced the outcomes of other subjects, or whether this influence also carried over to individual choice tasks in which the subjects' decisions influenced only their own outcomes. There is good reason to believe that eyes may influence decision-making in noninteraction tasks. A long line of psychological research has shown that the mere presence of others can facilitate the performance of simple tasks but impair the performance of more complex tasks (Bond \& Titus, 1983; Zajonc, 1965). With respect to choice behavior, research on accountability suggests that people care about how others view their decisions, even in individual choice tasks (Kruglanski \& Fruend, 1983; Lerner \& Tetlock, 1999; Vieider, 2011). In particular, when subjects know that their decisions will be made public, they adjust their behavior to comply with the prevailing view among their audience. If the view of the audience is unknown, the subjects engage in pre-emptive self-criticism, by carefully analyzing the problem to arrive at a more justifiable decision (Lerner \& Tetlock, 1999). These findings are intuitive as people are unlikely to be exclusively concerned with signaling a cooperative disposition; they will, for example, also care about appearing smart, conscientious, and successful. Therefore, if eye-like stimuli trigger a feeling of being monitored, their impact should not be limited to triggering pro-social behavior in interaction tasks, but can be expected to extend to individual choice tasks. 
However, it is not definite that the effect of eyes should extend beyond interaction tasks. Cosmides (1989) and Cosmides and Tooby $(1989,1992)$ argued that humans have evolved specialized, domainspecific cognitive modules for solving problems that are encountered in social exchange. To support this claim, the authors showed empirical evidence that a specialized cheater-detection mechanism existed. Later research suggested that people also have a memory bias for cheaters (see Mealey et al, 1996, Oda, 1997, and Oda \& Nakajima, 2010; see Barclay and Lalumière, 2006, and Mehl and Buchner, 2008, for contradictory findings). The ability to detect and remember cheaters may be necessary to successfully establish relationships of mutual cooperation. However, this ability is not sufficient because people must also aspire to cooperate in the first place. Kiyonari et al. (2000) therefore proposed the existence of a "social exchange heuristic," which facilitates the establishment of mutual cooperation by encouraging subjects to perceive one-shot prisoner dilemmas as assurance games in which mutual cooperation is the most preferable outcome. As argued by Oda et al. (2011), the eye effect may be due to a similar social heuristic that evolved to facilitate mutual cooperation. If this social heuristic is the cause, then there is no a priori reason to expect pictures of eyes to have any effect in the absence of interaction and thus, no reason to believe that eyes will influence behavior in individual choice tasks.

Secondly, in addition to exploring whether pictures of eyes influenced behavior in individual choice tasks, we investigated the nature of that influence by comparing this effect with the effect of another condition that was designed to remind the subjects of other people in their social group. The literature is somewhat ambivalent regarding whether eyes are special cues or simply one among many social cues that could produce the same result. For instance, in addition to presenting subjects with pictures of eyes, Haley and Fessler (2005) manipulated auditory cues that indicated the presence of others by using sound-deafening earmuffs. The authors found that the earmuffs appeared to reduce the subjects' generosity, although the effect did not reach statistical significance. Lambda and Mace (2010) studied whether the presence of other students influenced decisions in an ultimatum game if the subjects were explicitly guaranteed that their decisions would remain anonymous. The authors found that the presence of other students did not affect the subjects' behavior and cited this result as evidence against an eye effect. Being reminded of others without being exposed to a direct eye gaze may not have the same effect as an eye cue. To investigate whether the effects were the same, we also implemented a peers condition in which pictures of our subjects' social group (i.e., university students) were displayed during the experiment. 


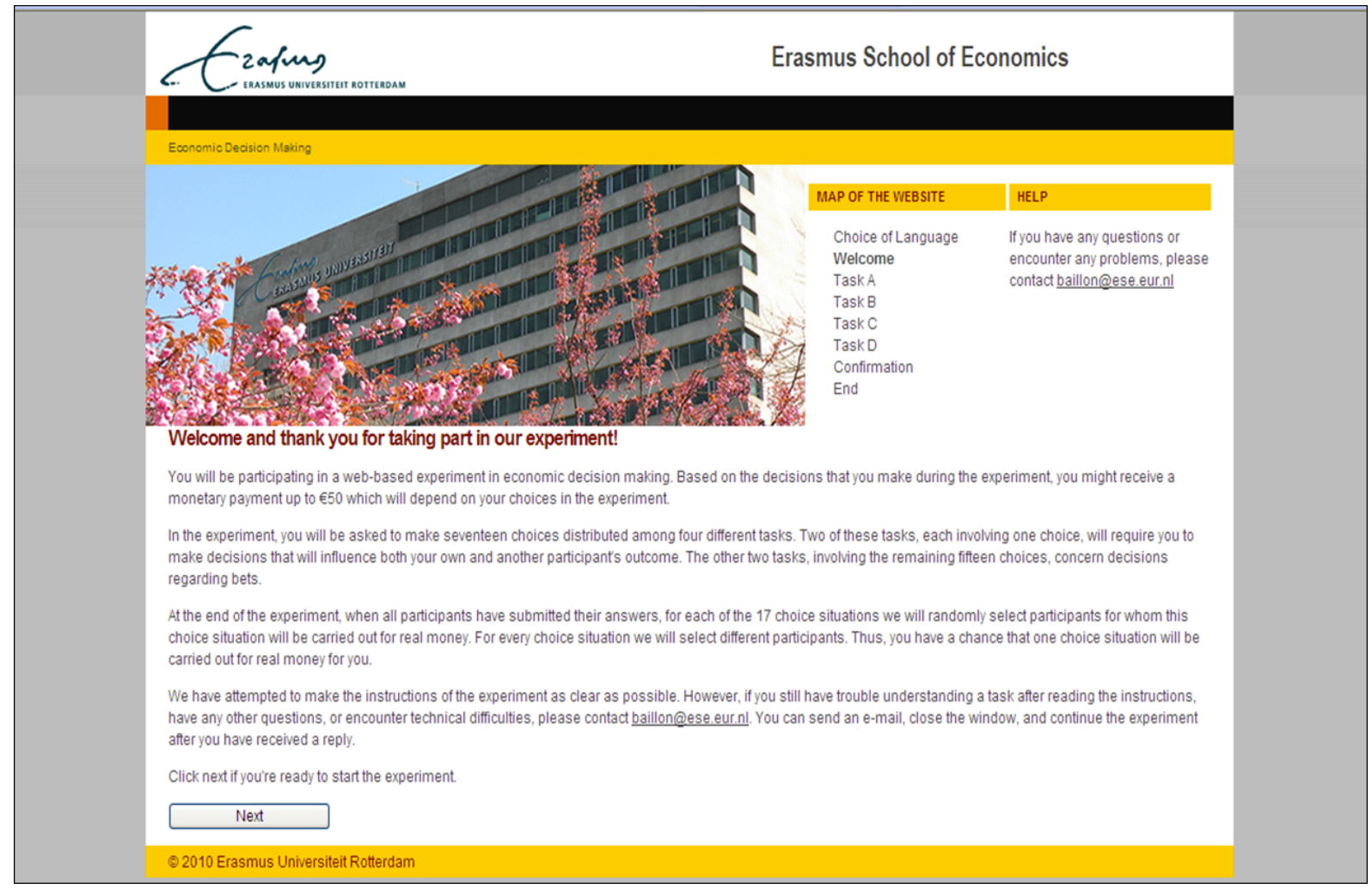

Figure 1: Screenshot of the university website as used in the experiment. Placed at the top left of the screen, the pictures randomly rotate every six seconds. The picture displayed on the screenshot above is one of the images that were common to all conditions.

\section{Method}

\subsection{Subjects}

We conducted an online experiment on 165 students from the Erasmus School of Economics (henceforth ESE), Erasmus University Rotterdam, the Netherlands (32\% females, age range = 18-33, mean $=21.1$ years, S.D. $=2.06$ years). The experiment was conducted during the first half of June 2010. We sent an email that contained personalized links to the website developed for the experiment to 600 students. The students were informed that the deadline to participate was two weeks after receipt of the recruitment email and that the payment for their participation could range up to $€ 50$; they received an email reminder one week after the initial email. The invitation emails and instructions can be found in the electronic supplementary material. The subjects were permitted to withdraw from the experiment at any time and their data were analyzed anonymously. 


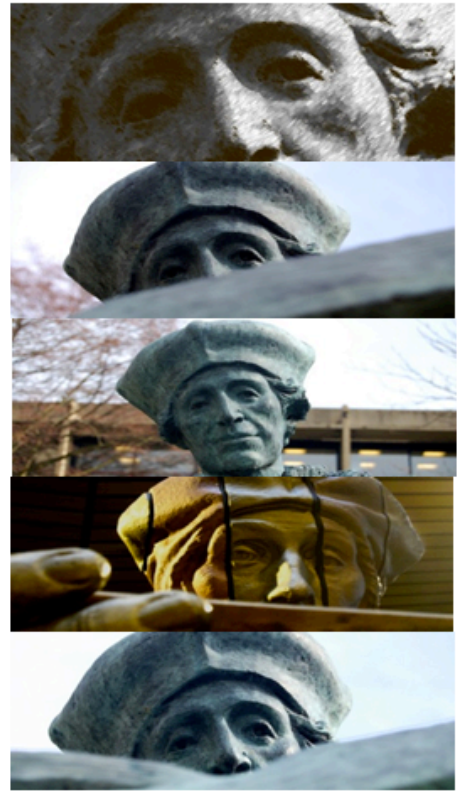

A

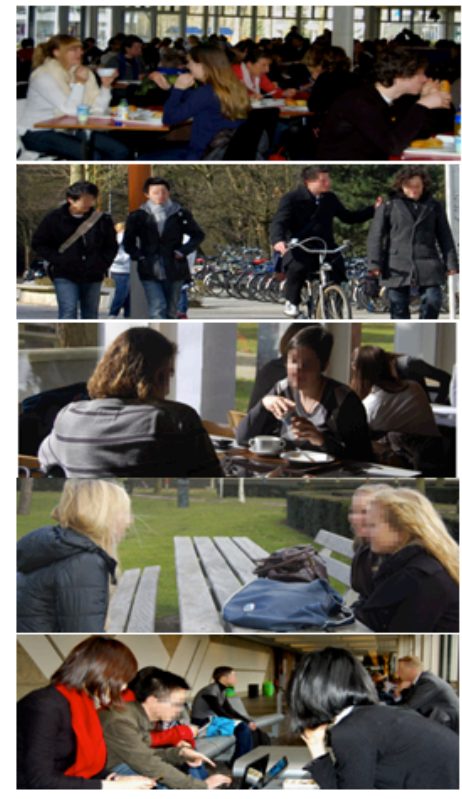

B

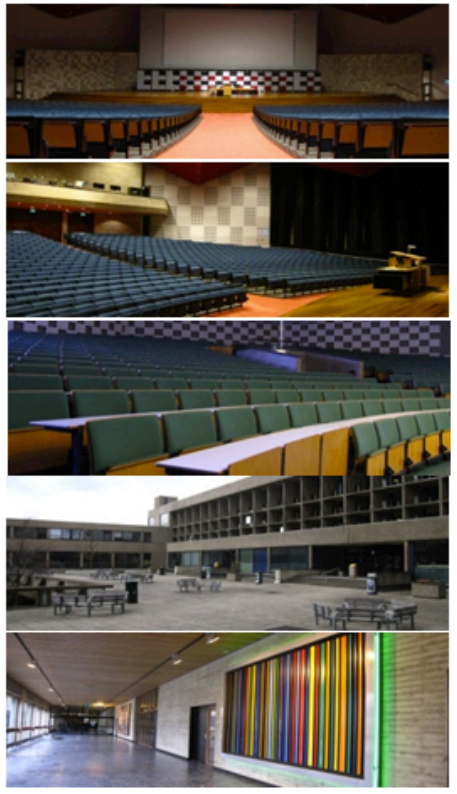

C

Figure 2. Pictures used in each condition. (A) Eyes, (B) Peers, (C) Control. (In the experiment, the faces of the people in the peers pictures were visible. The faces have been obscured here for publication purposes only.)

\subsection{Procedure}

We constructed a replica of the ESE website (Figure 1) for this experiment. After the initial login to any computer at the ESE, Internet Explorer opens up automatically. The homepage consists of the ESE website, which displays news and important information. Students and staff members are required to use this website to look up information and for many administrative procedures. Similarly to the ESE website, our experimental website was bilingual (Dutch and English) and compatible with most browsers (such as Internet Explorer, Mozilla Firefox, Opera, Safari, and Chrome) and most screen sizes.

To present our subjects with pictures of eyes and peers in an unobtrusive manner, we used the picture banner from the official ESE website. This banner typically displays rotating pictures from the campus. The pictures rotate randomly at an approximate interval of six seconds. We constructed three conditions by manipulating the types of pictures that rotated in this banner. The banner was visible to the subjects during the entire experiment.

For the eyes condition, we used pictures of the faces of statues of Erasmus, who is the school's namesake. The students are familiar with images of Erasmus because there are multiple statues of him on the campus and his image appears on official university documents. Thus, using such pictures would not appear out of the ordinary, and we could safely assume that the cues remained 
sufficiently subtle. Moreover, the neutral facial expressions displayed by the statues reduced the risk of accidently priming emotions (Figure $2 \mathrm{~A}$ ).

For the peers condition, we used pictures of students who were not looking directly at the camera to avoid a potential eye effect. The students in these pictures were engaged in studying, chatting, having lunch, etc., on campus. Our subject pool consisted of undergraduate students, thus the representations of their fellow university students could act as social cues that remind them of their own social group (Figure 2B, please note that faces have been obscured for publication purposes but were visible in the experiment).

Finally, as a control, we used pictures of empty halls from university buildings (Figure 2C). On the whole, the pictures from the three conditions did not differ much from pictures one could find on any university website and were similar to the pictures normally found on the ESE website. In addition to these condition specific pictures, the subjects also viewed two pictures of university buildings that were common to all conditions and were taken from the ESE website. Each subject was randomly allocated to one of the three conditions, and all of the tasks were carried out for real money for some randomly selected subjects after the experiment.

During the experiment, the subjects completed four tasks: two tasks involved interaction between the subjects, and two tasks involved individual choices under uncertainty. The order of the tasks was randomized across subjects. The four tasks were selected on the basis of past research and were designed so that social cues can be expected to impact the subjects' behavior. Each task and the corresponding predictions are described in detail below.

At the end of the experiment, the students answered a small questionnaire including demographic questions (gender, age, nationality, and education). For details we refer to the electronic supplementary material. Some of the answers for the first task described below were missing. Approximately 60 subjects were asked to re-enter their answers, of whom 12 failed to do so. As this affected every condition equally, there was no reason to believe that it would affect our results. We nonetheless studied whether it had any effect on our results and found that it had none (see electronic supplementary material). For each task, we report simple non-parametric tests for differences between conditions. The more advanced parametric statistical models that controlled for the subjects' characteristics are reported in the electronic supplementary material. All of the results reported in this paper were robust, and statistical significance was generally stronger in the more advanced analyses than in the simple analyses. 


\subsection{Task 1: Joy of destruction mini-game}

The first interaction task we used was the so-called Joy of Destruction mini-game (JoD) (Abbink \& Herrmann, 2010). Although research on cooperation and social-preferences has traditionally focused on pro-social behavior, a recent and growing body of literature has begun to apply economic games to the study of anti-social behavior, such as the anti-social punishment of cooperators in public good settings (e.g., Gächter \& Herrmann, 2009; Gächter et al., 2010; Herrmann et al., 2008). The JoD has been used in this literature to show that a considerable fraction of subjects is willing to pay money to destroy part of the payoff to another subject. In particular, the subjects destroyed their opponents' payoffs only infrequently when their behavior could be perfectly observed and their opponents could find out with certainty what caused the destruction. However, when the scenario was altered so that their opponent could no longer find out with certainty whether the destruction was caused by nature or by intention, the subjects' willingness to destroy markedly increased. Note that this difference occurred despite the complete anonymity of the subjects in both cases (Abbink \& Herrmann, 2010; Abbink \& Sadrieh, 2009).

To achieve a significant amount of destruction and thereby facilitate the investigation of possible differences between our conditions, we adopted the "hidden" setup of the JOD in which it is unclear to the subjects what caused the reduction of their income. In our JoD variant, two subjects each received an endowment of $€ 25$. Then, unaware of each other's identity, both subjects were asked whether they would be willing to pay $€ 1$ to destroy $€ 10$ of the other subject's endowment. There was a $1 / 3$ probability that $€ 10$ of the opposing subject's endowment would be destroyed regardless of the subject's decision, making it impossible for the opposing subject to tell what caused the destruction.

In the JoD game, there is no compelling rationale behind destruction: it is harmful to others and costly to oneself. Previous findings on the JOD further suggest that destruction mainly occurs in situations in which the behavior cannot be perfectly observed. In light of these findings, and of past studies that have showed that eyes increase pro-social behavior in simple tasks, we consider this task a way to validate whether the effect of our eyes cues align with the past findings of eyes. Furthermore, the design of this task also allows us to compare the effect of the eyes to the peers condition in an interaction task. 


\subsection{Task 2: Dictator game}

The second interaction task was the dictator game, which is widely studied in economics and which demonstrates what is often deemed to be pure altruism on the part of the subjects (Camerer, 2003). In this game, one subject, the dictator, received a monetary endowment of $€ 50$ and was asked how much she would donate to another anonymous subject. The other subject simply received what had been donated to her, and nothing else. The pro-social action here was to donate some money to the receiver, but this would in return lower the dictator's own income. We chose this task because the impact of eye-like stimuli on the dictator game has been studied before (Haley \& Fessler, 2005; Oda et al., 2011; Rigdon et al., 2009 Nettle et al., 2013). These past studies found that donation rates were significantly higher in response to eye cues. Including this task in our experiment thus provides us with another opportunity to see whether we could replicate the eye effect in our web-based setup. Furthermore, it provided us with a second opportunity to compare the effect of the eyes to the effect of peers in an interaction task.

\subsection{Task 3: Ellsberg's paradox}

The third task we employed was a variant of the standard ambiguity aversion task devised by Ellsberg (1961). The task included two bags containing black and red chips. In one bag (Bag K), the proportion of red and black chips was known, whereas in the second bag (Bag U), this proportion was unknown. The subjects were asked to choose a color (black or red) and a bag from which to draw a chip. If the color of the drawn chip matched the color that the subject had chosen, then the subject received $€ 50$.

When the proportion of red and black chips is 50-50, Bag K and Bag $U$ are normatively equivalent. Following Laplace's argument that ignorance should be represented by a uniform probability distribution, Bag $U$ should also be considered as a 50-50 bag. If the subjects do not follow this argument and believe that one of the colors makes up more than $50 \%$ of the balls in Bag $U$, then they should bet on this color and strictly prefer Bag U. Nevertheless, many studies have shown that a disproportionate number of people choose Bag K (Camerer \& Weber, 1992). The distaste for the unknown bag is often referred to as ambiguity aversion, and, given that the bags are normatively equivalent, can be interpreted as a bias (see, for instance, Raiffa, 1961).

In our experiment, we implemented the standard Ellsberg choice situation with a 50-50 proportion of red and black chips in Bag $\mathrm{K}$, however we also varied the proportion of red and black chips from 10\%$90 \%$ to $90 \%-10 \%$ (i.e., $10 \%-90 \%, 20 \%-80 \%, 30 \%-70 \%$...). For each possible proportion for Bag $\mathrm{K}$, the 
subjects were asked to state which bag ( $\mathrm{K}$ or $\mathrm{U})$ they would prefer to draw a ball from. When the probability was different from $50 \%$, the subjects overwhelmingly selected the normatively superior option, i.e., Bag $\mathrm{K}$ if the probability of winning in this bag was $60 \%$ or higher, and Bag $\mathrm{U}$ if the probability of winning in Bag K was $40 \%$ or lower. No clear differences between the conditions could therefore be detected in these scenarios (see electronic supplementary material). Hence, we report only our analysis of the traditional 50-50 case.

Previous studies have shown that being observed by others matters for this task. Curley et al. (1986) found that publicly experiencing the consequence of one's own decision in an Ellsberg task generates more ambiguity aversion compared to the situation where privacy was ensured (see also Muthukrishnan et al., 2009, and Trautmann et al., 2008). These authors argued that subjects will fear a negative evaluation if the bet's outcome is not in their favor, and the subjects will believe that choosing bag $\mathrm{K}$ is easier to justify due to its informational advantage (its content is known, unlike the one of bag U). Therefore, if our social cues (eyes and peers) trigger concerns of being monitored, we would expect more ambiguity aversion in those conditions compared with the control.

\subsection{Task 4: Simple vs. compound lotteries}

Bar-Hillel (1973) has shown that people show systematic biases when comparing simple gambles to compound gambles. To be more specific, people appear to overestimate the likelihood of conjunctive events (e.g., drawing, with replacement, four red chips from a bag with 10 black and 10 red chips) and underestimate the likelihood of disjunctive events (e.g., drawing, with replacement, at least one red chip from a bag with 9 black chips and 1 red chip when the subject is permitted four tries). The cause for this bias is often thought to be a realization of the anchoring and adjustment heuristic (Tversky \& Kahneman, 1974). It is believed that, when the subjects evaluate the compound event, they think about the probability of drawing a particular chip, which then takes the role of an anchor. If the subjects do not adjust properly for the compound nature of the event, then they overestimate conjunctive events and underestimate disjunctive events. Thus, people overvalue the conjunctive gambles and undervalue the disjunctive gambles.

In the final task, we investigated the effect of our cues on subjects' evaluation of compound gambles. The subjects were asked to make six choices between simple and conjunctive (compound) gambles. The options presented to the subjects were similar to the ones proposed by Bar-Hillel (1973) and have previously been implemented by Vieider (2011). For instance, in a simple gamble, a subject extracted one chip from a bag that contained 10 red and 10 black chips. The subject received $€ 50$ if 
the chip was red. In the conjunctive, compound gamble, the subject extracted 7 times (with replacement) from a bag that contained 18 red and 2 black chips. The subject won $€ 50$ if the chip was red each time. In all of the choice-situations of this task, the probability of winning in the simple gamble exceeded the probability of winning in the conjunctive, compound gamble. Although the simple gamble was thus objectively superior to the compound gamble, past research has showed that a significant number of people found the compound gamble more attractive (Bar-Hillel, 1973; Kruglanski \& Fruend, 1983; Vieider, 2011).

In line with the view that lowered anonymity leads to a desire to make better, more justifiable choices, Kruglanski and Freund (1983) and Vieider (2011) found that subjects who expected their choices to be evaluated later on were more likely to make the correct choice when deciding between simple and compound events. Therefore, if our social cues (eyes and peers) triggered the subjects' concerns of being monitored, we would expect them to make fewer mistakes in these conditions compared to the control.

\section{Results}

\subsection{Task 1: Joy of destruction mini-game}

The overall destruction rate obtained in the JoD over the three conditions was similar to the findings in Abbink \& Herrmann (2010). Over our entire sample, $24.84 \%$ of the subjects decided to destroy (N $=153$ ), compared with $25.8 \%$ of the subjects in Abbink \& Herrmann's (2010) experiment. Across conditions, however, we observed sharp differences.

In our control condition ( $N=51$ ), the subjects destroyed $38.78 \%$ of the time (Figure $3 \mathrm{~A}$ ). The destruction rate was halved in the eyes $(N=49)$ and peers $(N=53)$ conditions compared with the control condition, constituting a significant decrease (eyes: $17.65 \%, \chi 2(1)=5.534, P=0.019$; peers: $18.87 \%, \chi 2(1)=4.959, P=0.026)$. There was no significant difference between the eyes and the peers condition $(\chi 2(1)=0.026, P=0.872)$. 

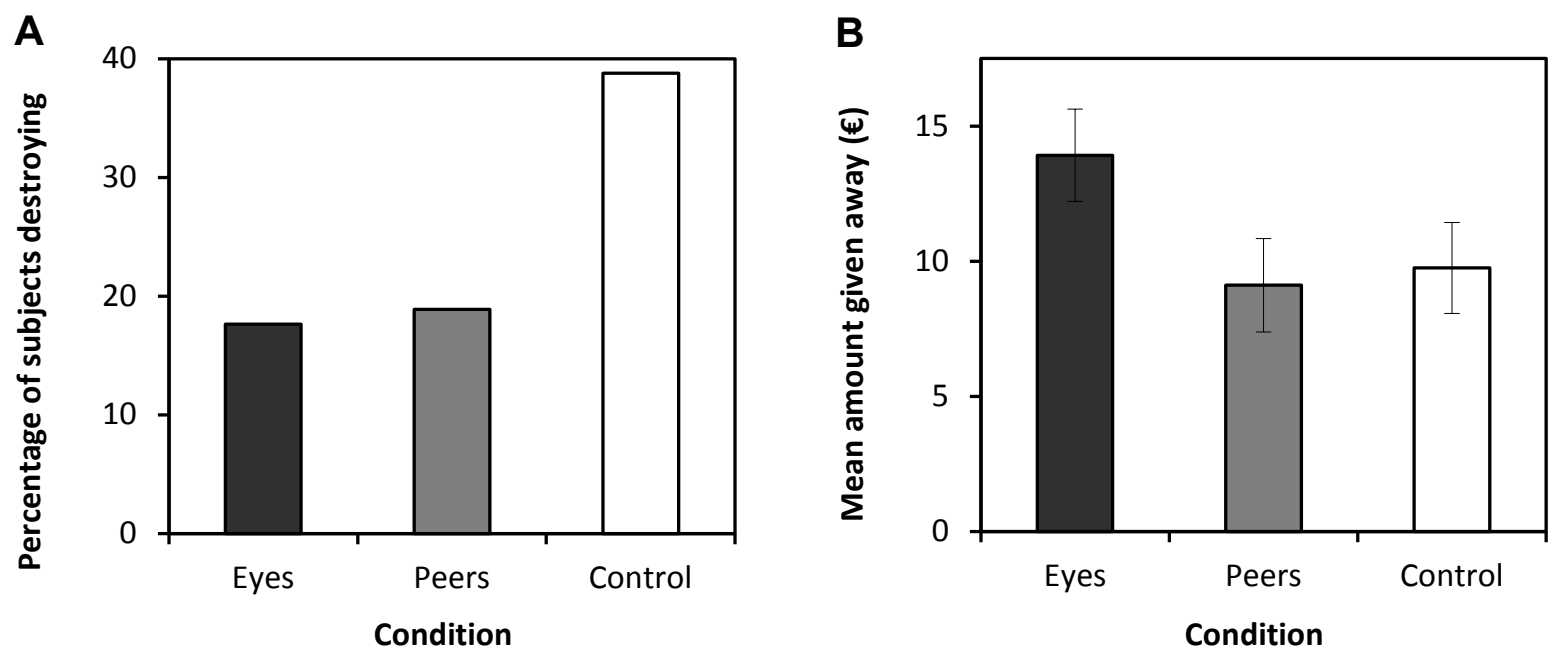

Figure 3: Results from the interaction tasks. The graph in (A) shows the percentage of subjects who chose to destroy their opposing subject's money in the JoD mini-game, while the graph in (B) shows the mean amount of money that was transferred in the dictator game for the different conditions. Error bars in the graph in (B) show \pm 1 standard error of the mean.

\subsection{Task 2: Dictator game}

The standard finding with respect to the dictator game is that over $60 \%$ of the subjects decide to give away money. The mean donation rate across all subjects is typically $20 \%$ of the endowment, although the rational, self-interested action is not to allocate any money to the other subject (Camerer, 2003). Across our entire sample, our findings were in line with the statistics presented above; a total of $63.64 \%$ of our subjects gave away money, while the average amount transferred was $€ 10.93$, or approximately $22 \%$ of the $€ 50$ endowment ( $N=165,55$ in each condition).

In our control condition, the subjects gave away $€ 9.75$ on average (Figure 3B). The pictures of eyes strongly increased donations to an average amount of $€ 13.93$ (Mann-Whitney, $z=-1.989, P=0.047$ ). By contrast, the average donation in the peers condition did not significantly differ from the control (mean: €9.11, Mann-Whitney, $z=0.817, P=0.414$ ). The donations amounts were significantly different between the eyes and the peers condition (Mann-Whitney, $z=-2.497, P=0.013$ ). 

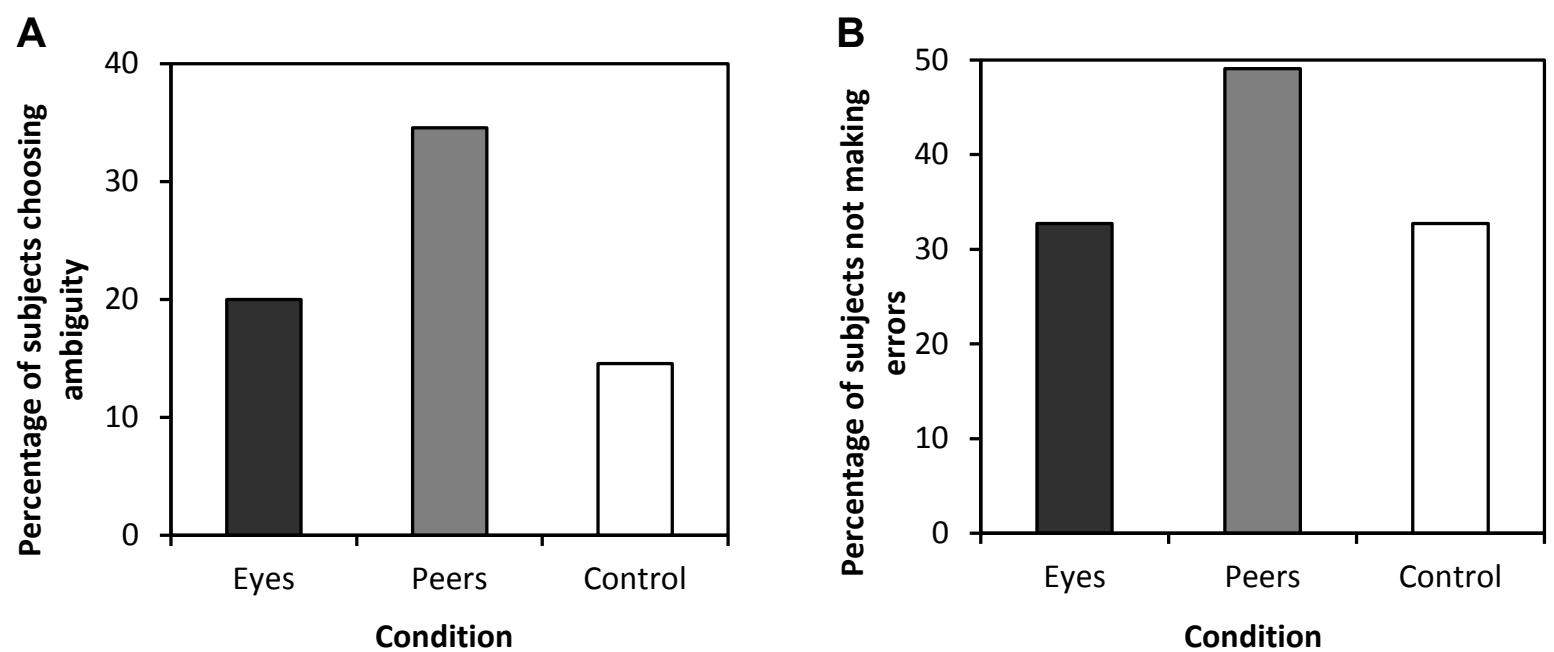

Figure 4: Results from the individual choice tasks. The graph in (A) shows the percentage of subjects who chose the ambiguous option (Bag U) over the risky option (Bag K), while the graph in (B) shows the percentage of subjects who did not make any errors in the final task.

Regarding the probability of donating, we found the highest rates of donation in the eyes condition, in which $76.36 \%$ of the subjects donated some amount. In the control, the percentage of subjects who donated was considerably lower than in the eyes condition, at $63.64 \%$, and the lowest rate of donation occurred in the peers condition, at 50.91\%. Here, however, neither the eyes nor the peers condition differed significantly from the control $(x 2(1)<2.121, P>0.145)$. The eyes and peers conditions differed significantly from each other, in that the subjects from the eyes condition were significantly more likely to donate compared the subjects in the peers condition $(\chi 2(1)=7.700, P=$ 0.006).

\subsection{Task 3: Ellsberg's paradox}

In the Ellsberg task, the subjects chose between two bags. The probability of winning was known for Bag K (50\%) and unknown for Bag U. In line with past findings, we observed that the majority of subjects chose Bag $\mathrm{K}$ in our control condition, while only a small fraction selected Bag $U(N=55$, $14.45 \%$, see Figure $4 A)$. In contrast to the interaction tasks, we found no effect of eyes on the subjects' bag choice $(\mathrm{N}=55,20 \%, \chi 2(1)=0.573, \mathrm{P}=0.449)$. In the peers condition, however, the subjects were significantly less likely to show a bias against the ambiguous option than in the other conditions: more than a third of the subjects in this condition chose Bag $U(N=55,34.55 \%$, comparison with the control: $\chi 2(1)=5.939, P=0.015$, comparison with the eyes: $\chi 2(1)=2.933, P=$ 0.087). 


\subsection{Task 4: Simple vs. compound lotteries}

The simple gamble is always preferable to the compound gamble; thus, we will refer to the choices that favor the compound gambles as errors. In the control condition $(N=55)$, fewer than a third of the subjects did not make any errors (Figure 4B). There was no difference between the eyes condition $(\mathrm{N}=55)$ and the control condition (both $32.73 \%, \times 2(1)=0.000, P=1$ ). In the peers condition $(N=55)$, however, $49.09 \%$ of the subjects never made an error. The difference between the peers condition and the two other conditions was marginally significant when the other conditions were separate (both: $\chi 2(1)=3.046, P=0.081$ ) and significant at the five percent level when the other two conditions were combined $(\chi 2(1)=4.160, P=0.041)$.

The number of errors reveals a similar pattern to the results presented above. The median number of errors made was one out of six in the peers condition, compared with two out of six in the other two conditions. The mean number of errors made was 2.27 in the control, 1.98 in the eyes condition and 1.60 in the peers condition. Mann-Whitney tests indicated that the difference in the number of errors was marginally significant between the peers and the control conditions (Mann-Whitney, $z=$ $1.766, P=0.077)$. The eyes condition did not differ significantly from the two other conditions $(P>$ $0.229)$.

\section{Discussion}

In the current paper, we applied a dual strategy to better understand the effect of pictures of eyes on human behavior. First, to identify whether the eye effect was limited to interaction tasks, we expanded the range of tasks to include individual choice tasks. Second, to ascertain whether eyes were special or were simply one among many social cues that may produce the same results, we compared the effect of eyes with the effect of another condition that presented the subjects with pictures of other students (peers).

In agreement with past findings, we found that pictures of eyes led to more pro-social behavior in interaction tasks. Our results revealed that the subjects gave more money to strangers and were less likely to destroy the endowment of others in response to eyes cues. However, we found that eyes did not influence subjects' behavior in individual choice tasks, in which the choices did not influence the outcomes of others. This difference suggests that the eye effect is limited to the situations that involved interaction, which is compatible with the view that this effect may be caused by a social exchange heuristic that works to establish mutual cooperation, as suggested by Oda et al. (2011). 
The differences between the eyes condition and the peers condition show that different social cues can have different behavioral implications. In the dictator game, the eyes promoted giving, while the peers did not. Moreover, the peers influenced behavior in the two individual choice tasks, while the eyes did not. The finding that different social cues can have different effects is important because it implies that care is required to avoid drawing overly general conclusions from the observed effects of one specific social cue.

It is noteworthy that, in the individual choice tasks, the peers condition uniformly increased economic rationality. In that condition, we observed less ambiguity aversion and fewer mistakes in choices between simple versus compound lotteries. In the interaction tasks, we found that peers only influenced behavior in the JoD game, where the pro-social act of not destroying is also economically rational. By contrast, peers did not appear to influence behavior in the dictator game, in which the pro-social and the rational action misalign. In short, the criterion of economic rationality seems to play an important role in the peers condition. It is possible that this effect may be an artifact of our subject pool, which consisted of subjects who were all trained in economics and might fear negative judgment from their peers if they do not make a rational decision. However, it should be noted that this finding also agrees with the general tenet of the accountability literature that considering the judgment of others will encourage pre-emptive self-criticism and careful analysis of the problem to arrive at a more justifiable decision (Lerner \& Tetlock, 1999). While the finding of the peers condition in the ambiguity task contradicts the recent literature that suggests that considering others' judgment will increase ambiguity aversion, it is important to note that these papers have all focused on the observation of the actual outcome by others. The accountability literature suggests that expecting judgment based on the outcomes of one's decisions generally hampers performance, while expecting judgment based on the decision process employed generally improves performance (Siegel-Jacobs and Yates, 1996; Simonson and Staw, 1992). It may be that being presented with pictures of peers during decision-making caused the latter, rather than the former, mechanism to operate. The latter mechanism could explain the results obtained in the current study.

It is possible that an alternative mechanism, different from considerations about others' judgment, may have caused the peers effect. For example, pictures that feature multiple people may trigger a competitive mindset, i.e., a desire to outperform others. Alternatively, the pictures in the peers condition, which displayed other people who did not look directly at the camera, may have made anonymity even more salient than the pictures in the control condition, which did not show any people at all. While the former explanation could account for the increased performance in individual choice tasks, it is not straightforward how the latter could do so. More importantly, both mechanisms fail to account for the findings in the interaction tasks. Competitive subjects should give 
less than other subjects in the dictator game, which we did not observe. Furthermore, both increased competitiveness and anonymity should be expected to increase destruction in the JoD game. In this game, subjects with a competitive mindset may attempt to improve their relative payoffs by destroying part of their opponents' endowment, and increasing anonymity has been found to increase destruction rates in previous studies (Abbink and Herrman, 2010; Abbink and Sadrieh, 2009). By contrast, we found that destruction was significantly lower in the peers condition compared with the control.

The influence of our subtle cues on the subjects' behavior is remarkable, given that the pictures we employed were common pictures that can be found on any university website. Furthermore, it is noteworthy that we found significant effects for both of the social cues in a web-based experiment. Web-based experiments have the advantage of diminishing the participation costs for subjects because they do not need to come to the laboratory and are free to participate at any time. Furthermore, these experiments allow subjects to make decisions in their natural environment. The obvious drawback is that the environment in which subjects make their decisions is less controlled than it would be in the laboratory. For our experiment, it was possible that subjects were in a public setting when they participated in the experiment, which could reduce the relative effectiveness of the social cues (Ekström, 2012; Ernest-Jones et al., 2011; Powell et al., 2012). Therefore, using a webbased design instead of a carefully controlled anonymous laboratory setting potentially lowered our chances of finding statistically significant effects (i.e., increased type II errors). That we found statistically significant effects of eyes in both interaction tasks and peers in both the individual choice tasks and one of the interaction tasks suggests that reduction in control was not a major problem in our experiment.

Interestingly, in another recent web-based study, Raihani and Bshary (2012) were unable to find an eye effect in a dictator game played online using Amazon Mechanical Turk (AMT). Our experiment differs from theirs in a number of ways, which makes it difficult to conclusively identify what caused the results to differ. Raihani and Bshary (2012) argued that interacting via AMT may have caused the subjects to feel truly anonymous and therefore be irresponsive to subtle social cues, similar to the argument put forth by Lambda and Mace (2010). This increased anonymity may explain the discrepancy between our findings and the findings from Raihani and Bshary (2012), as AMT ensures a larger degree of anonymity than our experimental setup. In our experiment, the subjects received personalized links to participate in the experiment and the payment of randomly selected subjects was conducted face-to-face so that the subjects could verify that the gambles in individual choice tasks were fairly resolved. Another explanation for the difference, however, may be that, in our experiment, the subjects played the game against fellow students from the same university, while 
the subjects in Raihani and Bshary's experiment played against subjects from all over the world. In light of Mifune et al.'s (2010) finding that pictures of eyes make people act more altruistically only towards members from their own in-group, this provides another explanation for why we found a significant effect of eyes while Raihani and Bshary (2012) did not.

To study the eye effect in an unobtrusive manner, we used pictures of Erasmus' eyes. Seeing Erasmus on the website would be normal for our subjects, who all studied at the Erasmus School of Economics. However, the image of a famous intellectual such as Erasmus could induce a desire to appear smart. Priming subjects with words such as "professor" has been found to improve subjects' performance at answering trivia questions (Dijksterhuis \& van Knippenberg, 1998). Nevertheless, we do not believe that our experiment was compromised in such a way. First, it should be noted that all of the subjects from the three conditions were, in a sense, primed with "Erasmus" because the name Erasmus was displayed at least four times on each screen for each condition (see Figure 1, at the top and at the bottom) and on the pictures that were common to all conditions. Moreover, the website that was used closely resembled that of the Erasmus School of Economics. Second, previous research showed that priming subjects with university-related concepts decreased the number of mistakes made by subjects (Dijksterhuis \& van Knippenberg, 1998). In our experiment, such priming should mean that subjects should have made fewer errors in the individual choice tasks in the eyes condition. As we have observed, especially in the choices between simple and compound gambles, this reduction in errors did not occur. Pictures of eyes did not lead to better decisions.

Observing that eyes do not influence behavior in our individual choice tasks does not guarantee that eyes will not influence behavior in any individual choice task. It could be argued that subjects react to pictures of eyes only when the task allows them to demonstrate positive qualities, such as being smart, conscientious, or successful, in an obvious manner and that our tasks did not allow them to do so. However, it is important to stress that both of the individual choice tasks were specifically selected to maximize the chance of observing an eye effect. For both tasks, past research indicates that manipulating anonymity in these tasks influences subjects' behavior. Thus, people appear to consider the judgment of others while performing these tasks. Moreover, in the task that compared simple vs. compound lotteries, qualities such as intelligence or conscientiousness could be demonstrated by choosing the objectively superior gamble (all of our subjects had attended mathematical courses about probability theory).

To conclude, our findings suggest that eyes should be considered distinct from other social cues, such as reminders of peers. Although reminders of peers influence a broad range of tasks, the eye effect appears to be limited to triggering pro-social behavior in situations that involve interaction. Combined with findings from previous studies, these results are in line with the claim that responses 
to eyes are caused by a social exchange heuristic aimed at enhancing cooperative behavior among ingroup members (Mifune et al., 2010), mediated by increased expectations of future reward (Oda et al., 2011). 


\section{References}

Abbink, K., \& Herrmann, B. (2010). The moral costs of nastiness. Economic Inquiry, 49, 631-633.

Abbink, K., \& Sadrieh, A. (2009). The pleasure of being nasty. Economics Letters, 105, 306-308.

Bar-Hillel, M. (1973). On the subjective probability of compound events. Organizational Behavior and Human. Performance, 9, 396-409.

Barclay, P. \& Lalumière, M.L. (2006). Do people differentially remember cheaters? Human Nature, $17,98-113$

Bateson, M., Nettle D., \& Roberts, G. (2006). Cues of being watched enhance cooperation in a realworld setting. Biology Letters, 2, 412-414.

Bond, C.F., \& Titus, L.J. (1983). Social facilitation: A meta-analysis of 241 studies. Psychological Bulletin, 94, 265-292.

Bourrat, P., Baumard, N., \& McKay, R. (2011). Surveillance Cues Enhance Moral Condemnation. Evolutionary Psychology, 9, 193-199.

Burnham, T.C., \& Hare, B. (2007). Engineering human cooperation. Does involuntary neural activation increase public goods contributions? Human Nature, 18, 88-108.

Camerer, C.F. (2003). Behavioral game theory: Experiments in strategic interaction. Princeton, NJ: Princeton University Press.

Camerer, C.F., \& Weber, M. (1992). Recent developments in modeling preferences: Uncertainty and ambiguity. Journal of Risk and Uncertainty, 5, 325-370.

Cosmides, L. (1989). The logic of social exchange: has natural selection shaped how humans reason? Studies with the Watson selection task. Cognition, 31, 187-276.

Cosmides, L., \& Tooby, J. (1989). Evolutionary psychology and the generation of culture: Part II. A computational theory of social exchange. Ethology and Sociobiology, 10, 51-97.

Cosmides, L., \& Tooby, J. (1992). Cognitive adaptations for social exchange. In: J. H. Barkow, L. Cosmides, \& J. Tooby (Eds.), The adapted mind: evolutionary psychology and the generation of culture (pp. 163-228). NewYork: Oxford Univ. Press.

Curley, S.P., Yates, J.F., \& Abrams, R.A. (1986). Psychological sources of ambiguity avoidance. Organizational Behavior and Human Decision Processes, 38, 230-256.

Dijksterhuis, A. \& van Knippenberg, A. (1998). The relation between perception and behavior, or how to win a game of trivial pursuit. Journal of Personality and Social Psychology, 74, 865-877.

Ekström, M. (2012). Do watching eyes affect charitable giving? Evidence from a field experiment. Experimental Economics, 15, 530-546.

Ellsberg, D. (1961). Risk, ambiguity, and the Savage axioms. Quarterly Journal of Economics, 75, 643669. 
Engelmann, D. \& Fischbacher, U. (2009). Indirect reciprocity and strategic reputation building in an experimental helping game. Games and Economic Behavior, 67, 399-407.

Ernest-Jones M., Nettle D., \& Bateson M. (2011). Effects of eye images on everyday cooperative behavior: a field experiment. Evolution and Human Behavior, 32, 172-178.

Fehr, E., \& Schneider, F. (2010). Eyes are on us, but nobody cares: are eyes cues relevant for strong reciprocity? Proceedings of The Royal Society B: Biological Sciences, 277, 1315-1323.

Gächter, S. \& Fehr, E. (1999). Collective action as a social exchange. Journal of Economic Behavior and Organization, 39, 341-369.

Gächter, S. \& Herrmann, B. (2009). Reciprocity, culture and human cooperation: Previous insights and a new cross-cultural experiment. Philosophical Transactions of The Royal Society B: Biological Sciences, 364, 791-806.

Gächter, S., Herrmann, B., \& Thöni, C. (2010). Culture and cooperation. Philosophical Transactions of The Royal Society B: Biological Sciences, 365, 2651-2661.

Haley, K.J., \& Fessler, D.M.T. (2005). Nobody's watching? Subtle cues affect generosity in an anonymous economic game. Evolution and Human Behavior, 26, 245-256.

Herrmann, B., Thöni, C., \&, Gächter, S. (2008). Antisocial punishment across Societies. Science, 319, 1362-1367.

Jaeggi, A.V., Burkhart, J.M., \& Van Schaik, C.P. (2010). On the Psychology of Cooperation in Humans and other Primates: Combining the Natural History and Experimental Evidence of Prosociality. Philosophical Transactions of The Royal Society B: Biological Sciences, 365, 2723-2735.

Kiyonari, T., Tanida, S., \& Yamagishi, T. (2000). Social exchange and reciprocity: confusion or a heuristic? Evolution and Human Behavior, 21, 411-427.

Kruglanski, A.W. \& Freund,T. (1983). The freezing and unfreezing of lay-inferences: Effects on impressional primacy, ethnic stereotyping, and numerical anchoring. Journal of Experimental Social Psychology, 19, 448-468.

Lamba, S., \& Mace, R. (2010). People recognise when they are really anonymous in an economic game. Evolution and Human Behavior, 31, 271-278.

Lerner, J.S. \& Tetlock, P.E. (1999). Accounting for the effects of accountability. Psychological Bulletin, $125,255-275$.

Mealey, L., Daood, C., \& Krage, M. (1996). Enhanced memory for faces of cheaters. Ethology and Sociobiology, 17, 119-128.

Mehl, B. \& Buchner A. (2008). No enhanced memory for faces of cheaters. Evolution and Human Behavior, 29, 35-41

Mifune, N., Hashimoto, H., \& Yamagishi, T. (2010). Altruism toward in-group members as a reputation mechanism. Evolution and Human Behavior, 31, 109-117. 
Milinsky, M., Semmann, D., Bakker, T.C.M., \& Krambeck, H.J. (2001). Cooperation through indirect reciprocity: image scoring or standing strategy? Proceedings of The Royal Society B: Biological Sciences, 268, 2495-2501.

Milinsky, M., Semmann, D., \& Krambeck, H.J. (2002). Reputation helps solve the 'tragedy of the commons'. Nature, 415, 424-426.

Muthukrishnan, A. V., Wathieu, L., \& Xu, A.J. (2009). Ambiguity aversion and the preference for established brands. Management Science, 55, ß1933-1941.

Nettle D., Harper Z, Kidson A, Stone R, Penton-Voak IS, Bateson M. (2013). The watching eyes effect in the Dictator Game: it's not how much you give, it's being seen to give something. Evolution and Human Behavior, 34, 35-40.

Oda, R. (1997). Biased face recognition in the Prisoner's Dilemma game. Evolution and Human Behavior, 18, 309-315.

Oda, R., \& Nakajima, S. (2010). Biased face recognition in the Faith Game. Evolution and Human Behavior, 31, 118-122.

Oda, R., Niwa, Y., Honma, A, \& Hiraishi, K. (2011). An eye-like painting enhances the expectation of a good reputation. Evolution and Human Behavior, 32, 166-171.

Powell, K.L., Roberts, G., Nettle, D. (2012). Eye Images Increase Charitable Donations: Evidence From an Opportunistic Field Experiment in a Supermarket. Ethology, 118, 1096-1011.

Raiffa, H. (1961). Risk, uncertainty and the Savage axioms: Comment. Quarterly Journal of Economics, $75,690-694$.

Raihani, N.J., \& Bshary, R. 2012. A positive effect of flowers rather than eye images in a large-scale, cross-cultural dictator game. Proceedings of The Royal Society B: Biological Sciences, 279, 35563564 .

Rege, M. \& Telle, K. (2004). The impact of social approval and framing on co-operation in public good situations. Journal of Public Economics, 88, 1625-1644.

Rigdon, M., Ishii, K., Watabe, M., \& Kitayama, M. S. (2009). Minimal social cues in the dictator game. Journal of Economic Psychology, 30, 358-367. .

Seinen, S. \& Schram, A. (2006). Social status and group norms: Indirect reciprocity in a repeated helping experiment. European Economic Review, 50, 581-602.

Siegel-Jacobs, K., \& Yates, J.F. (1996). Effects of procedural and outcome accountability on judgment quality." Organizational Behavior and Human Decision Processes, 65, 1-17.

Simonson, I., \& Staw, B.M. (1992). Deescalation strategies: A comparison of techniques for reducing commitment to losing courses of action. Journal of Applied Psychology, 77, 419-426.

Trautmann, S.T., Vieider, F.M., \& Wakker, P.P. (2008). Causes of ambiguity aversion: Known versus unknown preferences. Journal of Risk and Uncertainty, 36, 225-243. 
Tversky, A. \& Kahneman, D. (1974). Judgment under Uncertainty: Heuristics and Biases. Science, 185, 1124-1130.

Vieider, F.M. (2011). Separating real incentives and accountability. Experimental Economics, 14, 507518.

Wedekind, C. \& Milinski, M. (2000). Co-operation through image scoring in humans. Science, 288, 850-852.

Zajonc, R.B. (1965). Social Facilitation. Science, 149, 269-274. 